\title{
EVERY ITERATED MORPHISM YIELDS A co-CFL
}

\author{
Jean BERSTEL \\ L.I.T.P., Université Pierre et Marie Curie, 4 Place Jussieu, 75230 Paris, France \\ Communicated by L. Boasson \\ Received March 1985
}

The set of left factors of any infinite word generated by an iterated morphism is shown to be the complement of a one-counter context-free language. This is used to solve a problem stated by Main, Bucher and Haussler (1985).

Keywords: Iterated morphism, context-free language, permutation-free word

\section{Introduction}

Recently, Main [3] and Main, Bucher and Haussler [4] disproved quite a lot of conjectures about various classes of context-free languages by showing that the complement of the set of left factors of the Thue-Morse sequence and of other related infinite words is context-free. The aim of this article is to extend the latter result by showing, with almost the same proof technique, that, for any iterated morphism, the complement of the set of left factors of an infinite word it generates is context-free. It appears in the construction that the language is even one-counter (in the sense of Greibach [2]).

\section{Notation}

Let $\mathrm{A}$ be an alphabet. A morphism $\mathrm{h}: \mathrm{A}^{*} \rightarrow \mathrm{A}^{*}$ is prolongeable in $\mathrm{a} \in \mathrm{A}$ if $\mathrm{h}(\mathrm{a})=\mathrm{au}$ for some nonempty word $u$. In this case, each $h^{n}(a)$ is a left factor of $h^{n+1}(a)$. If the lengths of these words are unbounded, then the morphism $h$ defines an infinite word

$x=a_{0} a_{1} \ldots a_{n} \ldots \quad\left(a_{i} \in A\right)$,

completely characterized by the condition that each $\mathrm{h}^{\mathrm{n}}$ (a) is a left factor of $\mathbf{x}$. The word $\mathbf{x}$ is also denoted by $h^{\omega}(a)$. It is called the infinite word generated by $\mathrm{h}$ at a. The morphism $\mathrm{h}$ itself is called an iterated morphism if there is a letter a for which it generates an infinite word.

Observe that the word $x=h^{\omega}$ (a) is completely determined by the following conditions:

$\mathrm{a}_{0}=\mathrm{a}, \quad \mathrm{h}(\mathbf{x})=\mathbf{x}$.

Also, observe that the words $h^{\mathrm{n}}$ (a) have in fact strictly increasing length, and, moreover, that for each left factor $u$ of $\mathbf{x}$,

$|\mathbf{h}(\mathbf{u})|>|\mathbf{u}|$.

Indeed, consider the integer $\mathrm{p}$ such that

$\left|h^{\mathrm{p}}(\mathrm{a})\right| \leqslant|\mathrm{u}|<\left|\mathrm{h}^{\mathrm{p}+1}(\mathrm{a})\right|$.

Then,

$|\mathrm{h}(\mathrm{u})| \geqslant\left|\mathbf{h}^{\mathrm{p}+1}(\mathrm{a})\right|>|\mathrm{u}|$.

\section{Result}

Theorem. Let $\mathrm{h}: \mathrm{A}^{*} \rightarrow \mathrm{A}^{*}$ be a morphism that generates an infinite word at a letter $\mathrm{a} \in \mathrm{A}$, and let $\mathrm{F}$ be the set of left factors of this infinite word. Then $\mathrm{A}^{*}-\mathrm{F}$ is one-counter context-free. 


\section{Proof. Set}

$\mathbf{x}=h^{\omega}(a)=a_{0} a_{1} \ldots a_{n} \ldots \quad\left(a_{n} \in A\right)$.

Since $h(x)=x$, each $h\left(a_{0} a_{1} \ldots a_{n}\right)$ is a left factor of $\mathbf{x}$. More precisely, $\mathbf{x}$ is defined inductively as follows:

(1) $a_{0}=a$,

(2)

$$
\begin{aligned}
& a_{k+1} \ldots a_{k+r}=h\left(a_{n}\right) \\
& \text { with } r=\left|h\left(a_{n}\right)\right|, \\
& k=\left|h\left(a_{0} a_{1} \ldots a_{n-1}\right)\right| .
\end{aligned}
$$

We shall describe a PDA that accepts a word $w=b_{0} b_{1} \ldots b_{m}$ iff

(i) $b_{0} \neq a_{0}$,

(ii) there exists an integer $\mathrm{i}<\mathrm{m}$ such that

$\left|h\left(b_{0} b_{1} \ldots b_{i-1}\right)\right|=k<m$,

and

$b_{k+1} \ldots b_{k+r}$ is a left factor of $h\left(b_{i}\right)$

with $r=\min \left\{\left|h\left(b_{i}\right)\right|, m-r\right\}$.

The check of condition (i) is easy. Also, statement ( $(2)$ is easily verified by a finite state memory. The only interesting point is the computation of the length of

$h\left(b_{0} b_{1} \ldots b_{i-1}\right)$.

The PDA guesses the integer $i$, and then, for each of the letters $b_{k} \quad(0 \leqslant k \leqslant i-1)$, it pushes $\left|h\left(b_{k}\right)\right|-1$ marks on the stack. (If $h\left(b_{i}\right)$ is the empty word, then the PDA pops. If the stack is empty, then the PDA stops in an unsuccessful state.) Thus, after reading $b_{0} b_{1} \ldots b_{i-1}$, there are

$\left|h\left(b_{0} b_{1} \ldots b_{i-1}\right)\right|-i$

marks on the stack. (Observe that if the guessed integer is $\mathrm{i}=0$, then the PDA immediately passes to the next phase.)

In the second phase, the PDA remenbers $b_{i}$ and then reads input letters, one for each mark in the stack. If there are not enough letters to empty the stack, the computation fails. Otherwise, the process stops when the bottom symbol is encountered. It is easily seen that, at this stage, the total number of input letters read is

$\left|h\left(b_{0} b_{1} \ldots b_{i-1}\right)\right|$.
Now the final test starts. Remembering that the guessed letter is $b_{i}$, the PDA reads available input, at most $\left|h\left(b_{i}\right)\right|$ letters, and checks that the word read is not a left factor of $h\left(b_{i}\right)$. In this case, the word is accepted and the computation halts.

It is clear that for a word in $F$ there is no accepting computation. Conversely, if a word $w$ is not in $F$, then either it does not start with $a_{0}$, or there is a longest word $u$ such that $u$ and $h(u)$ are left factors of both $w$ and $x$. But then, setting $w=a_{0} a_{1} \ldots a_{t} w^{\prime}$ and $u=a_{0} a_{1} \ldots a_{s}$, the PDA will accept $w$ when guessing the letter $a_{s+l}$. This completes the proof of the Theorem.

\section{An application to permutation-free words}

An abelian square (or permuted square) is a nonempty word of the form $x y$ where $x$ and $y$ are equal up to the order of the letters. A word is permutation-free if none of its factors is an abelian square. An infinite permutation-free word over a five-letter alphabet is given by Pleasants [5], no such word exists for three letters, and the question for four letters remains open. Main, Bucher and Haussler ask whether there is any context-free language with an infinite, permutation-free complement. We give a positive answer to this question.

Corollary. There exists a context-free language over a five-letter alphabet with an infinite, permutationfree complement.

Proof. A slight modification of a construction of Pleasants shows that there is an iterated morphism generating an infinite, permutation-free word. Let $A=\{a, b, c, d, e\}$ and let $s: A^{*} \rightarrow A^{*}$ be the isomorphism defined by the cyclic permutation $s(a)$ $=\mathrm{b}, \mathrm{s}(\mathrm{b})=\mathrm{c}, \ldots, \mathrm{s}(\mathrm{e})=\mathrm{a}$. The morphism

$\mathrm{h}: \mathrm{A}^{*} \rightarrow \mathrm{A}^{*}$

defined by

$h(a)=$ aebedebecedecea,

$\mathrm{h} \circ \mathrm{s}=\mathrm{s} \circ \mathrm{h}$,

has the property that $h^{\omega}(a)$ is permutation-free [5]. Thus, the Corollary follows from the Theorem. 
Remark. A counting argument easily shows that there exist infinite words $x$ such that the language $L(x)=\{w \mid w$ is not a left factor of $\mathbf{x}\}$

is not context-free. The following explicit example is from Grazon [1]. For the infinite word

$\mathbf{x}=a^{2} b a^{2^{2}} b \ldots a^{2^{\left(2^{n)}\right.}} b \ldots$,

the language $L(x)$ is not context-free. The proof is difficult because of the quite remarkable observation, also due to Grazon [1], stating that any language of the form $L(x)$ satisfies Ogden's iteration lemma for context-free languages.

\section{References}

[1] A. Grazon, Thèse de troisième cycle, June 1985.

[2] S. Greibach, An infinite hierarchy of context-free languages, J. Assoc. Comput. Mach. 16 (1969) 91-106.

[3] M.G. Main, An infinite square-free co-CFL, Inform. Process. Lett. 20 (2) (1985) 105-107.

[4] M.G. Main, W. Bucher and D. Haussler, Applications of an infinite square-free co-CFL, 12th ICALP, Nafplion, July 1985.

[5] P.A.B. Pleasants, Nonrepetitive sequences, Proc. Camb. Phil. Soc. 68 (1970) 267-274. 